\title{
Adaptive genetic differentiation in Pterocarya stenoptera (Juglandaceae) driven by multiple environmental variables were revealed by landscape genomics
}

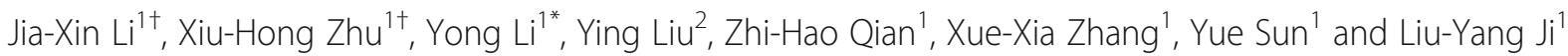

\begin{abstract}
Background: The investigation of the genetic basis of local adaptation in non-model species is an interesting focus of evolutionary biologists and molecular ecologists. Identifying these adaptive genetic variabilities on the genome responsible can provide insight into the genetic mechanism of local adaptation.

Results: We investigated the spatial distribution of genetic variation in 22 natural populations of Pterocarya stenoptera across its distribution area in China to provide insights into the complex interplay between multiple environmental variables and adaptive genetic differentiation. The Bayesian analysis of population structure showed that the 22 populations of $P$. stenoptera were subdivided into two groups. Redundancy analysis demonstrated that this genetic differentiation was caused by the divergent selection of environmental difference. A total of 44 outlier loci were mutually identified by Arlequin and BayeScan, 43 of which were environment-associated loci (EAL). The results of latent factor mixed model analysis showed that solar radiation in June (Sr6), minimum temperature of the coldest month (Bio6), temperature seasonality (Bio4), and water vapor pressure in January (Wvp1) were associated with the highest numbers of EAL. Sr6 was associated with the ecological habitat of "prefered light", and Bio6 and Wvp1 were associated with the ecological habitat of "warm and humid environment".
\end{abstract}

Conclusions: Our results provided empirical evidence that environmental variables related to the ecological habitats of species play key roles in driving adaptive differentiation of species genome.

Keywords: Adaptive genetic differentiation, Environment-associated loci, Genome scans, Landscape genomics, Pterocarya stenoptera

\section{Background}

Recently, the investigation of the genetic basis of local adaptation in non-model species has become an interesting focus of evolutionary biologists and molecular ecologists $[1,2]$. Locally adapted species are facing selection pressures from temporal climate fluctuations and spatial environment heterogeneity. In response to these selective pressures, species will undergo adaptive changes in phenotypes and phenology [3]. Behind these phenotypic and phenological changes is the

\footnotetext{
*Correspondence: liyongrui1@126.com

${ }^{\dagger}$ Jia-Xin Li and Xiu-Hong Zhu contributed equally to this work. ${ }^{1}$ Innovation Platform of Molecular Biology, College of Forestry, Henan Agricultural University, No.95, Wenhua Road, Zhengzhou 450002, China Full list of author information is available at the end of the article
}

adaptive differentiation of genes on the genome. Genome scans enable us to identify these adaptive genes responsible for local adaptation using population genetic analyses. Identifying the genes that control these phenotypic and phenological changes can provide insight into the genetic mechanism of local adaptation [4]. However, the identification of these adaptive genes on the genome responsible for local adaptation remains a great challenge for most non-model species due to the lack of genomic information [5].

Landscape genomics was proposed by Joost et al. [6]. It has been used to uncover the relationship between adaptive genes on the genome and heterogeneous environment variables among natural populations of 
species [7]. Although most non-model species have no genomic information, molecular markers that do not need prior information and have high density coverage of the genome are suitable for landscape genomic studies of non-model species. Thus, amplified fragment length polymorphisms (AFLPs), inter-simple sequence repeats (ISSRs), and start codon targeted polymorphisms (SCoTs) all conform to the above conditions [4]. Simultaneously, these markers also have the advantages of high polymorphism and high repeatability. However, AFLP and ISSR are neutral molecular markers; the adaptive loci detected by these markers are more likely to be linked to adaptive genes. SCoT is a molecular marker developed based on the short conserved initial codon; it is a non-neutral bias marker that is more biased toward genes [8]. Therefore, it is more suitable for adaptive evolution research compared with the other two markers. In recent years, several reduced-representation genome sequencing (RRGS) technologies have been developed to improve the genome coverage density of molecular markers. These sequencing technologies include genotyping by sequencing [9], restricted site associated DNA [10], and specific-locus amplified fragment sequencing [11]. Although these markers yielded by the RRGS are different from the three molecular markers mentioned above, they contain DNA sequence information. Most of them cannot be annotated because they are in non-coding regions on the genome or the DNA sequence is too short and there is no whole-genome information. In any case, they can give some information about those genes that can be annotated. Recently, landscape genomics studies using these molecular markers have been carried out on many plant and animal species [4].

A large number of landscape genomic studies have proved that environmental variables would drive the adaptive differentiation of some loci on the genome of locally adapted species $[12,13]$. However, we do not know why adaptive differentiation occurred in these genes and why environmental factors played key roles in driving adaptive differentiation. During local adaptation, different species in different regions have different adaptive differentiation genes and different driving factors. Are there common reasons behind these differences? Several recent landscape genomic studies have proposed a hypothesis that environmental variables related to ecological habitats play key roles in driving species adaptive evolution; in other words, the genes associated with these environmental variables will undergo adaptive differentiation [2, 14, 15]. More landscape genomic research is needed to test whether this hypothesis applies to other species as well. Another question worthy of discussion is whether divergent selection on the genome from environmental variables plays a decisive role on the spatial genetic structure of species. Given that previous surveys on species population structure used more neutral molecular markers, we pay more attention to the effects of population demographic history and gene flow on them [16]. If the whole genome markers are used, that is, both neutral and non-neutral markers, the genetic differentiation that driven by natural selection from environmental variables will be detected. Population demographic history, gene flow, and natural selection, which will have a greater impact on the spatial genetic structure of species? The answers to these questions will help deepen our understanding of adaptive evolution of species.

Pterocarya stenoptera C. DC (Juglandaceae) is a deciduous broad-leaved tree growing in forests below $1500 \mathrm{~m}$ above sea level along the stream bank or wet hillside land. It is widely distributed in warm temperate and subtropical zones of China. Pterocarya stenoptera prefers light, tolerates waterlogging, likes to grow in warm and humid environment, and can grow on acidic to slightly alkaline soil [17]. Here, 22 natural populations of $P$. stenoptera across its distribution region in China were sampled to investigate the relationship between adaptive genetic variations on the genome and environmental variables by using landscape genomic approach.

In this study, we employed SCoT markers to scan the genome of $P$. stenoptera and identified the adaptive loci by performing correlations between local environmental variables and selected SCoT alleles. The present study aimed to (i) identify the spatial genetic structure of $P$. stenoptera, (ii) evaluate the role of environmental variations on the spatial genetic structure of $P$. stenoptera, and (iii) examine the effects of environmental variables on adaptive differentiation of $P$. stenoptera genome.

\section{Results \\ Population genetic structure}

A total of 510 individuals of P. stenoptera from 22 wild populations were successfully scored using the $9 \mathrm{SCoT}$ primers, and 1006 unambiguous fragments were identified with sizes varying from $100 \mathrm{bp}$ to $1200 \mathrm{bp}$. The number of alleles in 9 primers ranged from 53 (SCoT35) to 156 (SCoT25). The lowest number and percentage of polymorphic alleles $\left(N_{\mathrm{A}}=126, P P A=\right.$ 12.5) were found in AHXN (P5) population and the highest $\left(N_{\mathrm{A}}=261, P P A=25.9\right)$ in JSBH $(\mathrm{P} 16)$ population. Nei's genetic diversity $\left(H_{\mathrm{E}}\right)$ per population varied from 0.0459 in AHXN (P5) to 0.083 in HNTM (P20). Overall, the summary statistics of the genetic diversity analyses of 22 populations of $P$. stenoptera are shown in Table 1.

The Bayesian analysis of the population structure of P. stenoptera (Fig. 1) demonstrated that the highest Delta $K$ value (Fig. 2) was obtained when 22 
Table 1 Details of population locations, sample size, genetic diversity of 22 populations for Pterocarya stenoptera

\begin{tabular}{|c|c|c|c|c|c|c|c|}
\hline Population no. and code & Locations & Altitude meters) & Lat.N)/ Long.E) & $N$ & $N_{\mathrm{A}}$ & $P P A$ & $H_{E}$ \\
\hline \multicolumn{8}{|l|}{ Group A } \\
\hline 1. JSLM & Lang Mt., Jiangshu & 53 & $31.95 / 120.89$ & 22 & 181 & 18.0 & 0.072 \\
\hline 2. SCWD & Wuduzhen, Sichuan & 532 & $31.88 / 104.78$ & 24 & 178 & 17.7 & 0.070 \\
\hline 3. HNJG & Jigong Mt., Henan & 418 & $31.81 / 114.08$ & 23 & 170 & 16.9 & 0.069 \\
\hline 4. AHTZ & Tianzhu Mt., Anhui & 39 & $30.67 / 116.49$ & 24 & 171 & 17.0 & 0.058 \\
\hline 5. ZJTM & Tianmu Mt., Zhejiang & 202 & $30.28 / 119.46$ & 22 & 183 & 18.2 & 0.061 \\
\hline 6. AHXN & Xiuning, Anhui & 155 & 29.78/ 118.17 & 24 & 126 & 12.5 & 0.045 \\
\hline 7. SCEM & Emei Mt., Sichuan & 533 & $29.57 / 103.44$ & 23 & 156 & 15.5 & 0.058 \\
\hline 8. JXSQ & Shanqing Mt., Jiangxi & 171 & 28.84/ 118.04 & 23 & 153 & 15.2 & 0.048 \\
\hline 9. JXLH & Longhu Mt., Jiangxi & 47 & $28.12 / 116.97$ & 24 & 180 & 17.9 & 0.062 \\
\hline 10. GZFJ & Fengjing Mt., Guizhou & 489 & 27.84/ 108.77 & 22 & 181 & 18.0 & 0.072 \\
\hline 11. YNYB & Yangbi, Yunnan & 1445 & $25.62 / 100.03$ & 23 & 170 & 16.9 & 0.069 \\
\hline \multicolumn{8}{|l|}{ Group B } \\
\hline 12. SDTM & Tai Mt., Shandong & 304 & $36.22 / 117.12$ & 24 & 139 & 13.8 & 0.054 \\
\hline 13. SDMM & Meng Mt., Shandong & 323 & $35.56 / 117.96$ & 24 & 156 & 15.5 & 0.062 \\
\hline 14. HNNZ & Nanzhao, Henan & 616 & $33.59 / 112.18$ & 24 & 201 & 20.0 & 0.067 \\
\hline 15. HNXC & Xichuan, Henan & 383 & $33.28 / 111.12$ & 20 & 216 & 21.5 & 0.079 \\
\hline 16. SXWZ & Wuzi Mt., Shaanxi & 408 & $32.95 / 107.84$ & 21 & 204 & 20.3 & 0.078 \\
\hline 17. JSBH & Baohua Mt., Jiangshu & 189 & $32.14 / 119.09$ & 24 & 261 & 25.9 & 0.077 \\
\hline 18. HBSN & Shengnongjia, Hubei & 657 & $31.37 / 110.50$ & 24 & 194 & 19.3 & 0.070 \\
\hline 19. HBJG & Jiugong Mt., Hubei & 74 & $29.45 / 114.71$ & 24 & 199 & 19.8 & 0.064 \\
\hline 20. HNTM & Tianmeng Mt., Hunan & 165 & $29.11 / 110.46$ & 24 & 231 & 23.0 & 0.083 \\
\hline 21. FJWY & Wuyi Mt., Fujian & 185 & $27.65 / 117.97$ & 24 & 178 & 17.7 & 0.065 \\
\hline 22. HNHM & Heng Mt., Hunan & 198 & 27.26/ 112.72 & 23 & 204 & 20.3 & 0.074 \\
\hline
\end{tabular}

populations were clustered into two groups. The first group is Group A (P1 to P11), and the second group is Group B (P12 to P22). Despite the 22 populations of $P$. stenoptera could be divided into two groups, the genetic variations between the two groups was very low $\left(6.69 \%, F_{\mathrm{CT}}=0.067, P<0.001\right.$; Table 2$)$, and most genetic variations occurred within populations $(75.67 \%$,
$F_{\mathrm{ST}}=0.243, P<0.001 ;$ Table 2$)$. The value of gene flow $(\mathrm{Nm})$ among all populations was 2.022 . To detect the roles of the 30 environmental variables in this genetic differentiation and their relative contribution on this differentiation, a constrained linear ordination analysis, redundancy analysis (RDA), was performed. The results of RDA are shown in Table 3 and Fig. 3.

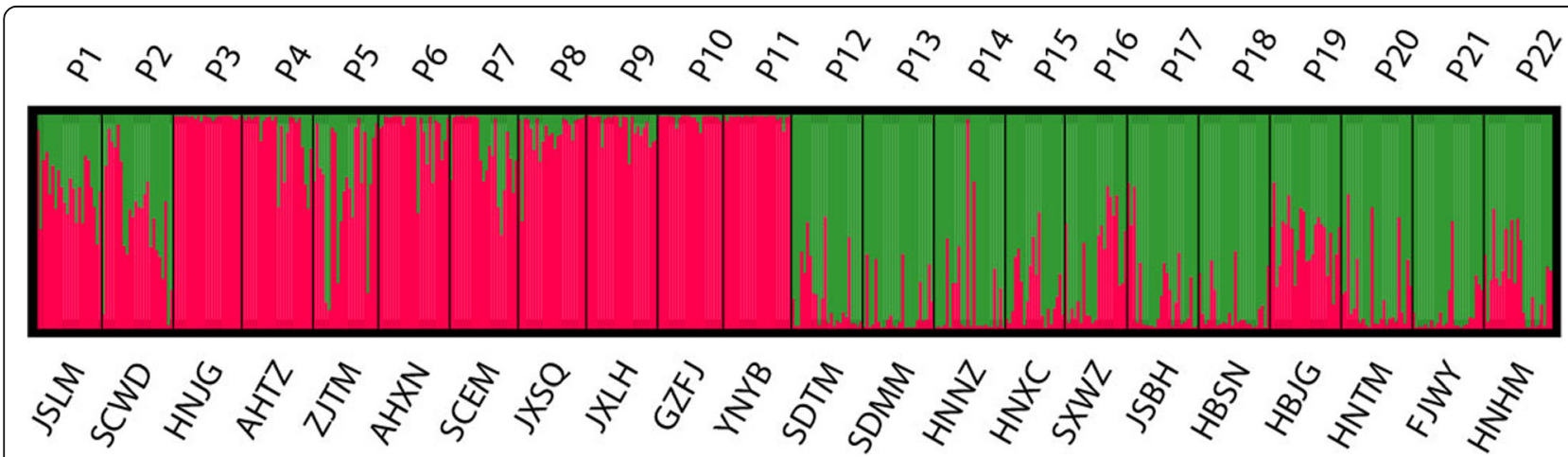

Fig. 1 STRUCTURE analyses of 22 sampled populations of Pterocarya stenoptera. Each vertical bar shows the proportional representation of two genetic clusters (K) for an individual 


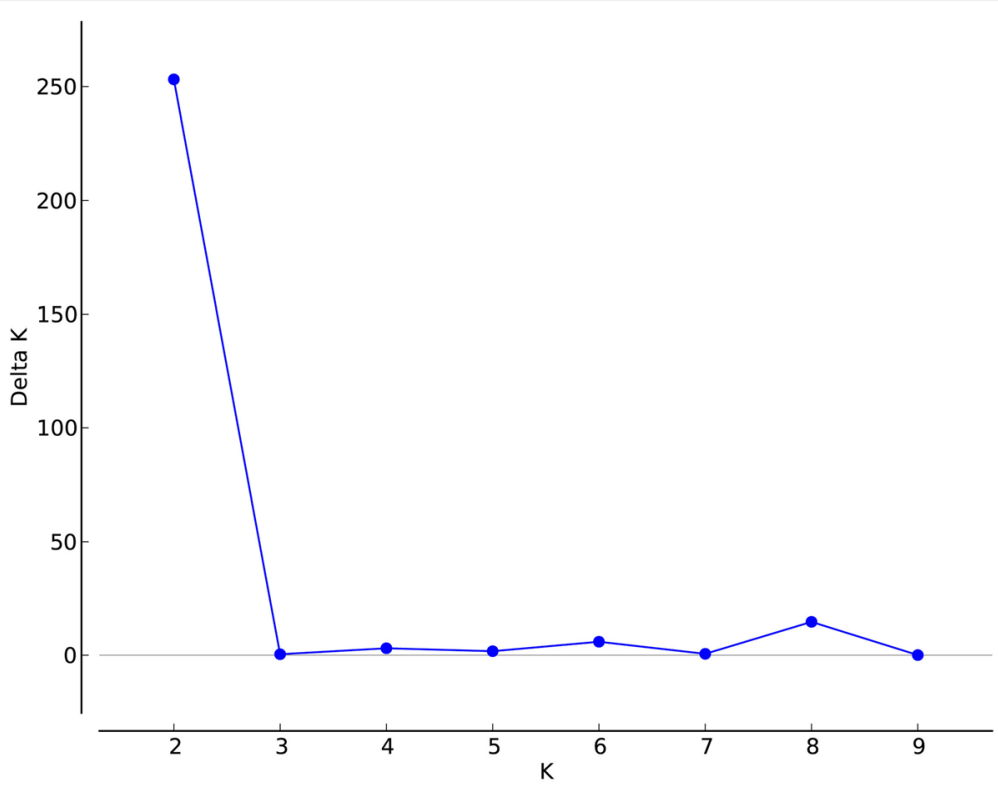

Fig. 2 The uppermost hierarchical level of population genetic structure determined according to the values of $\Delta K$. $\Delta K$ was calculated by Structure Harvester

Correlations between the genetic variables of 1006 alleles and the 25 environmental variables in axes 1 and 2 were both 1.000 . The ratios of the eigenvalues of axes 1 and 2 were 27.4 and $12.0 \%$, respectively. RDA analysis showed that these environmental variables could divide the populations of $P$. stenoptera into two groups, whereas the genetic differentiation between them is very weak (Fig. 3). This is consistent with the result of STRUCTURE and analysis of molecular variance (AMOVA). Five environmental variables were significantly correlated with RDA axes 1 and 2 (Table 3). Among these five environmental variables, mean diurnal range (Bio2), temperature seasonality (Bio4), and minimum temperature of the coldest month (Bio6) were related to temperature; solar radiation in June (Sr6) was related to light; and water vapor pressure in January (Wvp1) was related to air humidity. Bio6 and Sr6 showed the strongest correlation with genetic variables among the five environmental variables.

\section{Characterization of environment-associated loci (EAL)}

A total of 81 outlier loci (8.1\% of $1006 \mathrm{SCoT}$ alleles) with $F_{\mathrm{ST}}$ $P$-value below 0.05 were identified by using the hierarchical

Table 2 Hierarchical AMOVAs for SCOT variation surveyed in Pterocarya stenoptera

\begin{tabular}{lllll}
\hline Source of variation & d.f. & \%Total variance & F-statistic & $P$-value \\
\hline Among groups & 1 & 6.69 & $F_{\text {CT }}=0.067$ & $P<0.001$ \\
Among populations & 20 & 17.64 & $F_{\text {SC }}=0.189$ & $P<0.001$ \\
within groups & & & & \\
Within populations & 485 & 75.67 & $F_{\text {ST }}=0.243$ & $P<0.001$ \\
\hline
\end{tabular}

island model in Arlequin (Fig. 4; Additional file 1). Moreover, 168 outlier loci (16.7\% of 1006 SCoT alleles) with posterior probability above 0.76 (i.e., $\log 10 \mathrm{PO}>$ 0.5) were identified by using Bayesian method in BayeScan (Fig. 4; Additional file 1). To reduce the false positive rate, the loci detected by both methods were considered as outlier loci. Here, a total of 44 mutual loci (4.4\% of 1006 SCoT alleles) were detected by both methods. Latent factor mixed model (LFMM) analysis was subsequently performed to verify whether these outlier loci were driven by environmental variables. As a result, $43 \mathrm{EAL}$ (4.0\% of $1006 \mathrm{SCoT}$ alleles) associated with at least one environmental variable were identified (Table 4). Among the 25 environment variables that we detected, Sr6, Bio6, Bio4, and Wvp1 were associated with the highest numbers of EAL. The results suggested that they play major role in the genetic differentiation of $P$. stenoptera.

\section{Discussion}

In this study, we investigated the spatial distribution of genetic variation in wild populations of $P$. stenoptera across its distribution area in China to provide insights into the complex interplay between multiple environmental variables and adaptive genetic differentiation of this widespread broad-leaved tree species and thus improve our understanding of the genetic mechanism of local adaptation. Landscape genomics has developed rapidly in the past decade, which has been proved to be an effective method for studying adaptive evolution of species [4]. Here, we revealed the adaptive evolution 
Table 3 Correlations between environmental variables and the ordination axes

\begin{tabular}{|c|c|c|c|c|}
\hline $\begin{array}{l}\text { Environmental } \\
\text { variable }\end{array}$ & Axe 1 & Axe 2 & Axe3 & Axe 4 \\
\hline Bio1 & 0.330 & 0.226 & -0.137 & 0.184 \\
\hline $\mathrm{BiO} 2$ & -0.238 & $-0.440^{*}$ & -0.091 & 0.033 \\
\hline Bio3 & 0.208 & -0.200 & 0.043 & 0.102 \\
\hline Bio4 & $-0.422^{*}$ & -0.101 & -0.059 & -0.166 \\
\hline Bio5 & -0.036 & -0.011 & -0.116 & 0.099 \\
\hline Bio6 & $0.481^{*}$ & 0.245 & -0.005 & 0.205 \\
\hline Bio8 & -0.119 & 0.149 & 0.171 & $-0.457^{*}$ \\
\hline Bio9 & 0.338 & 0.100 & -0.102 & $0.429^{*}$ \\
\hline Bio12 & 0.317 & 0.160 & -0.098 & $0.440^{*}$ \\
\hline Bio13 & 0.184 & 0.124 & -0.253 & 0.290 \\
\hline Bio14 & 0.272 & 0.088 & 0.082 & $0.467^{*}$ \\
\hline Bio15 & -0.284 & -0.013 & -0.286 & -0.204 \\
\hline Bio18 & 0.281 & 0.252 & -0.103 & 0.055 \\
\hline Sr1 & 0.084 & -0.381 & 0.303 & 0.087 \\
\hline $\mathrm{Sr} 3$ & -0.044 & -0.382 & 0.295 & -0.140 \\
\hline Sr5 & -0.332 & -0.402 & 0.171 & -0.267 \\
\hline Sr6 & $-0.496^{*}$ & -0.338 & -0.021 & -0.195 \\
\hline Sr7 & -0.239 & -0.081 & -0.039 & 0.359 \\
\hline Sr9 & -0.052 & -0.177 & 0.123 & $0.512^{*}$ \\
\hline Sr10 & -0.090 & -0.341 & 0.258 & 0.292 \\
\hline Sr11 & 0.148 & -0.342 & 0.319 & 0.253 \\
\hline Wvp1 & $0.439^{*}$ & 0.363 & 0.033 & 0.375 \\
\hline Wvp4 & 0.316 & 0.309 & -0.075 & $0.415^{*}$ \\
\hline Wvp7 & 0.057 & 0.157 & 0.188 & 0.156 \\
\hline Wvp9 & 0.273 & 0.218 & 0.174 & 0.304 \\
\hline
\end{tabular}

Statistically significant correlation by ${ }^{*} P<0.05$ and ${ }^{* *} P<0.01$

of $P$. stenoptera in response to environmental variables by using $1006 \mathrm{SCoT}$ alleles.

The role of environmental factors in shaping the spatial genetic structure of species has been a key issue in landscape genomics research $[18,19]$. Spatial genetic structure of species is the result of the interaction of multiple factors, e.g., population demographic history, geographical or ecological barriers, transmission mode of seeds and pollen, geological events, and divergent selection of environmental factors $[2,20]$. In previous studies on population genetics, more attention was paid to the effects of the first few factors on the population genetic structure [21]. However, the efficient gene flow of species tends to obscure previous genetic structures, especially those based on nuclear genes [16]. Meanwhile, a completely new population genetic structure will be formed because of the divergent selection, genetic drift, and inbreeding. Although genetic drift and inbreeding can also rapidly alter the genetic structure of species, they occur more often in small and isolated populations [22]. However, P. stenoptera seems not suitable for this situation, due to a large population size. Meanwhile, there is high gene flow among populations of $P$. stenoptera $(N m=2.022)$. The efficient gene flow $(N m>1)$ would avoid the isolation between populations [23]. Therefore, the current genetic structure for $P$. stenoptera based on SCoT marker can hardly be attributed to genetic drift and inbreeding. Our survey showed that the 22 populations of $P$. stenoptera were divided into two groups, i.e., Group A (P1 to P11) and Group B (P12 to P22). Three possible reasons can be used to explain this intraspecific population differentiation. The first is caused by population demographic history. There were two refugia for $P$. stenoptera during the Quaternary glacial period; the present spatial genetic pattern was formed by the spread and redistribution of the population from the two refugia after glaciation. The second is due to geographical or ecological barriers. There is a geographical or ecological barrier between the two groups, which blocks or interferes with the gene flow and leads to the genetic differentiation between the two groups. The third is caused by divergent selection of environmental factors. The two groups are in different habitats, and different environmental factors lead to the allele frequency difference of the naturally selected genes, which eventually leads to the genetic differentiation between the two groups. Assuming that the first reason is true for $P$. stenoptera, the populations as refugia have the highest genetic diversity, and the population that is away from the refugia will gradually reduce its genetic diversity because of the founder effect. However, neither Group A nor Group B has gradient descent from the population with the highest genetic diversity. More importantly, Group A is discontinuous, which is separated by Group B. Thus, it is impossible to have two refugia for P. stenoptera. Overall, the first possible reason does not explain the genetic differentiation between the two groups of $P$. stenoptera. The second possible reason is considered to explain the genetic differentiation between the two groups of $P$. stenoptera. There must be ecological or geographical barriers between the two groups. In fact, the populations of the two groups are continuously distributed, and there are no geographical or geographical barriers. Even if the differentiation is caused by the second possible reason, the differentiation between them must be significant. Our results of hierarchical AMOVA $\left(F_{\mathrm{CT}}=0.067, P<0.001\right)$ do not support this interpretation. By assuming the genetic divergence of $P$. stenoptera caused by the third possible reason, a weak genetic differentiation between the two groups would be expected because of the interaction between 


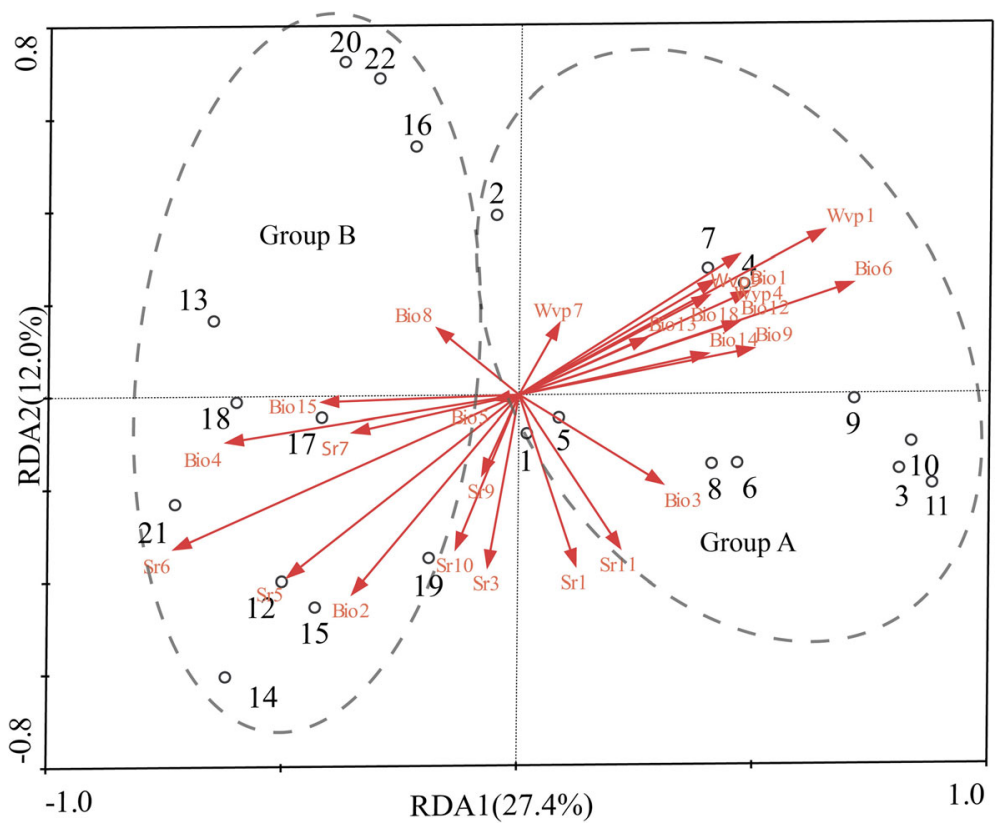

Fig. 3 Redundancy analysis of Pterocarya stenoptera showing the relative contribution of each environmental variation shaping population genetic structure. The biplot depicts the eigenvalues and lengths of eigenvectors for the RDA

strong gene flow and divergent selection of environmental factors. The results of hierarchical AMOVA were consistent with this expectation. Our RDA results (Fig. 3) also showed that environmental variables could slightly separate the two groups of P. stenoptera. The populations of Group A live at higher temperature of the coldest month and higher water vapor pressure in January than those of Group B, whereas populations of Group B live at higher value of temperature seasonality and higher solar radiation in June than those of Group A. The genetic differentiation caused by the divergent selection of environmental differences will be maintained due to the existence of heterogeneous environments, which is different from the genetic differentiation caused

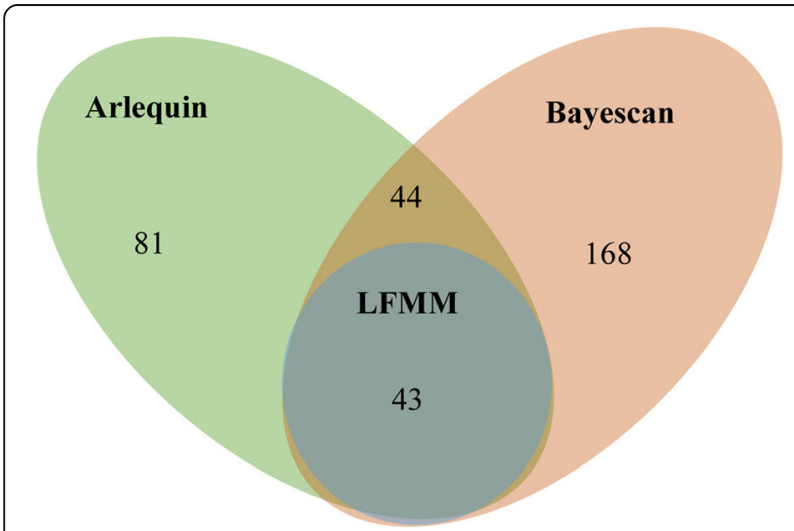

Fig. 4 The results of outlier loci detected by Bayescan and Arlequin and EAL were identified by latent factor mixed model analysis by genetic drift. Taken together, the third possible reason is more suitable for $P$. stenoptera.

Because of the inherent limitations of SCoT markers, sequence information cannot be obtained [4]. Thus, the genes cannot be annotated for their function. Although we do not know what genes these loci are or which genes they are linked to, we can know which environmental factors these loci are related to. Here, the results of LFMM analysis showed that Sr6, Bio6, Bio4, and Wvp1 were associated with the highest numbers of EAL, which suggested that these environmental variables play a major role on the genetic differentiation of $P$. stenoptera genome. Recent landscape genomic studies have proposed a hypothesis that environmental variables related to ecological habitats of species play key roles in driving adaptive differentiation of species genome [2, 14, 15]. Among the four environmental variables associated with the largest number of adaptive loci, Sr6 was associated with the ecological habitat of "prefered light", and Bio6 and Wvp1 were associated with the ecological habitat of "warm and humid environment". P. stenoptera blooms from April to May, and its fruits ripen from August to September. Therefore, we speculated that $P$. stenoptera might be more sensitive to light during fruit development. Our RDA results indicate that there is a significant difference in the amount of solar radiation in June (Sr6) between the two groups, which promoted the adaptive differentiation of these related adaptive loci. Bio6 and Wvp1 refer to the minimum temperature of the coldest 


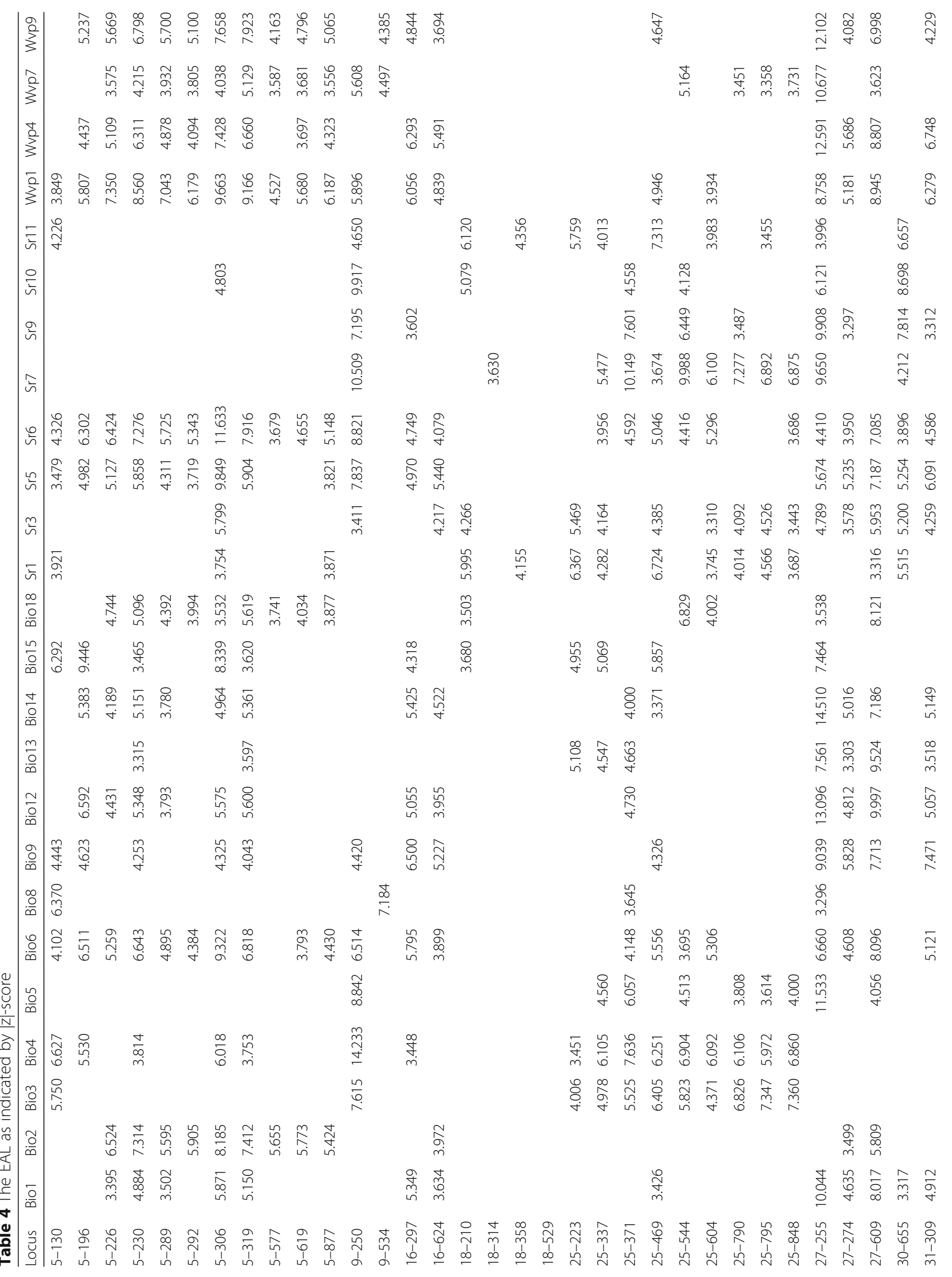




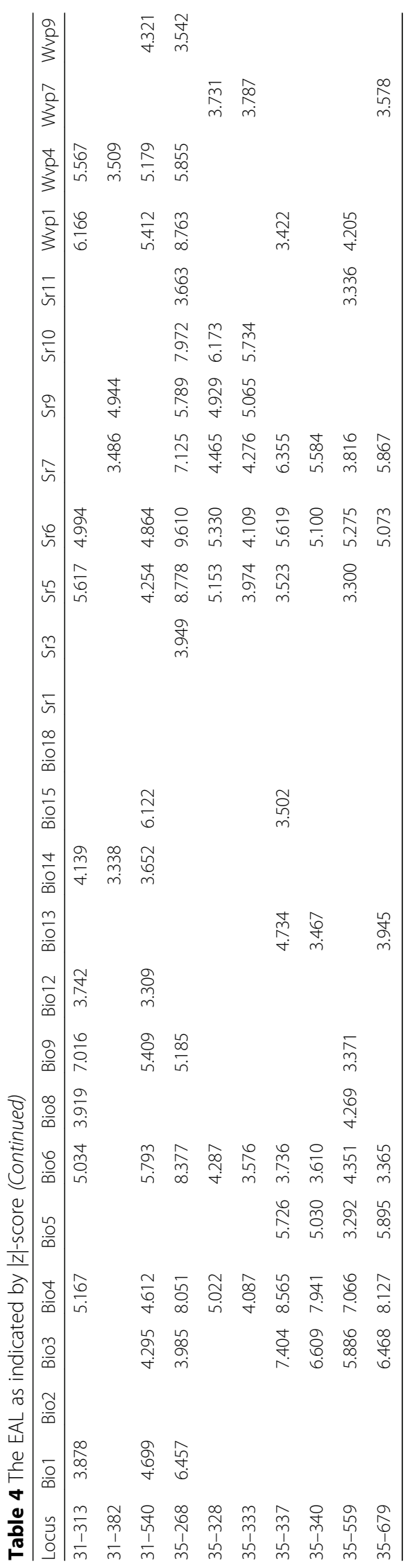


month and water vapor pressure in January, respectively. Similarly, there are significant differences of the two environmental variables between the two populations. In China, January is the driest month. These two variables were associated with larger numbers of adaptive loci, suggesting that temperature and water vapor in extreme environments were the main causes of their adaptive differentiation. In fact, previous studies have proved that there is a significant difference in the cold resistance of $P$. stenoptera from different provenance [24]. Surprisingly, seasonal variations in temperature (Bio4) might also have a significant impact on the adaptive differentiation of $P$. stenoptera. Overall, the hypothesis that environmental variables related to ecological habitats of species play key roles in driving adaptive differentiation of species genome is also suitable for P. stenoptera.

\section{Conclusions}

In the present study, SCoT markers were used to investigate adaptive genetic differentiation in $P$. stenoptera. Our survey showed that the 22 populations of $P$. stenoptera were divided into two groups. Although spatial genetic structure of species is the result of the interaction of multiple factors, our results suggested that the divergent selection of environmental differences play a major role on the genetic differentiation of P. stenoptera. Our results also provided empirical evidence that environmental variables related to ecological habitats of species play key roles in driving adaptive differentiation of species genome.

\section{Methods}

\section{Sample collection}

A total of 510 individuals from 22 natural populations were sampled from the entire distribution range of $P$. stenoptera in China (Fig. 5). Each population sample contained 20 to 24 individuals (Table 1), and each individual was at least $20 \mathrm{~m}$ away from each other. Young, healthy leaves were collected and stored in zip-lock bags containing silica gel at room temperature until DNA extraction. The geographical coordinates and number of samples for each population are shown in Table 1. After identified by Dr. Yong Li, each population deposit a voucher specimen at the herbarium of College of Forestry, Henan Agricultural University, Zhengzhou, China (voucher no. LiPS2017001-2,017,022). No specific permits were required for $P$. stenoptera, all samples were collected following current Chinese regulations.

\section{Molecular protocols}

Genomic DNA was extracted from approximately 30 $\mathrm{mg}$ of dried leaves by using the standard Plant DNA Extraction Kit (Tiangen, Beijing, China) protocol.

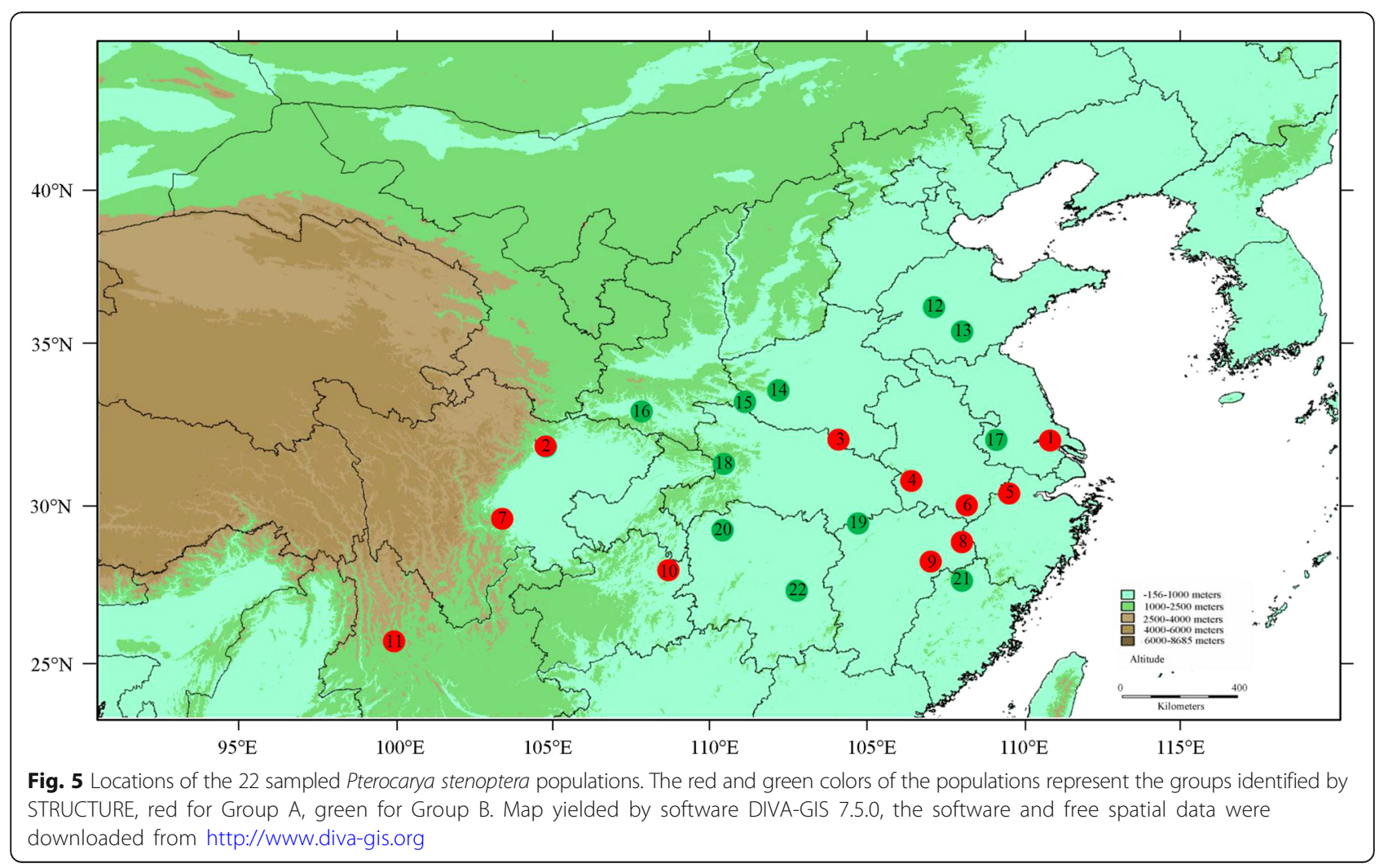


DNA was then quantified using the ND5000 Ultra Micro UV-Vis spectrophotometer (BioTeke, Beijing, China). All individuals were genotyped using SCoT markers. Despite its inherent defects lacking DNA sequence information, the SCoT marker has been used for landscape genomic studies because it has the advantages of non-requirement for genomic information, high repeatability, and high throughput [2, 14, 15]. After preliminary screening of the polymorphism and reproducibility of all SCoT primers [25], nine primers (SCoT5, SCoT9, SCoT16, SCoT18, SCoT25, SCoT27, SCoT30, SCoT31, and SCoT35) were selected for polymerase chain reactions (PCRs). SCoT5, SCoT16, and SCoT25 were 5' FAM fluorescently labeled primers; SCoT9, SCoT18, and SCoT30 were 5' HEX primers; SCoT27, SCoT31, and SCoT35 were 5' TAMRA primers. PCR amplification was carried out in a $20 \mu \mathrm{L}$ reaction mixture containing $20 \mathrm{ng}$ of template DNA, 10 $\mathrm{mM}$ reaction buffer ( $\mathrm{pH} \mathrm{8.3),} 0.2 \mathrm{mM}$ of each dNTP, $0.3 \mu \mathrm{M}$ primer, and 1 unit of Taq polymerase (Tiangen, Beijing, China). PCRs were performed in a Mastercycler nexus thermocycler (Eppendorf, Hamburg, Germany) with an initial denaturation at $94{ }^{\circ} \mathrm{C}$ for 5 min followed by 35 cycles with denaturation at $94^{\circ} \mathrm{C}$ for $40 \mathrm{~s}$, primer-specific annealing temperature $\left(52^{\circ} \mathrm{C}\right.$ for SCoT27; $56^{\circ} \mathrm{C}$ for SCoT5, SCoT9, SCoT16, SCoT18, SCoT30, SCoT31, and SCoT35; and $58^{\circ} \mathrm{C}$ for SCoT25) for $40 \mathrm{~s}$, extension at $72^{\circ}$ $\mathrm{C}$ for $90 \mathrm{~s}$ and with a final extension at $72{ }^{\circ} \mathrm{C}$ for $5 \mathrm{~min}$, and termination by a final hold at $4{ }^{\circ} \mathrm{C}$. Finally, $3 \mu \mathrm{L}$ of PCR products was mixed with $10 \mu \mathrm{L}$ of $\mathrm{HiDi}$ formamide and electrophoresed on an ABI 3730 DNA Analyzer at BGI (Beijing, China). The size of PCR products is determined according to the internal standard LIZ1200 (Applied Biosystems, Foster City, USA).

\section{Data analysis}

The SCoT fragments were identified based on the presence or absence of peaks viewed in GeneMarker 2.2.0 (SoftGenetics, State College, Pennsylvania, USA). The raw information was then transformed into a $1 / 0$ matrix. To reduce the error reading rate of SCoT fragments, the peaks within $100-1200 \mathrm{bp}$ and relative fluorescent units above 200 were scored. Subsequent population genetic analyses were carried out with the basis of the 1/0 matrix from SCoT markers.

Genetic parameters, including polymorphic allele number $\left(N_{\mathrm{A}}\right)$, allele frequencies, Nei's measure of gene diversity $\left(H_{\mathrm{E}}\right)$ [26], and percentage of polymorphic alleles $(P P A)$, were calculated using AFLP-SURV 1.0 [27]. Genetic structure of the 22 populations of $P$. stenoptera was assessed using the Bayesian-based program STRUCTURE 2.3.4 [28]. The program was run with $K$ values from 1 to 10 with 10 replicates for each $K$, and a burn-in period of 10,000 and 10,000 Markov chain Monte Carlo iterations. The admixture model with independent allele frequencies was used for this analysis. The optimal $K$ value with the most suitable population clusters was judged according to the $\Delta K$ values introduced by Evanno et al. [29], and this method was executed by Structure Harvester [30]. The average value of the admixture coefficients over 10 runs was calculated using CLUMPP 1.1 [31]. The barplots of STRUCTURE were obtained by DISTRUCT 1.1 [32]. The distribution of genetic differentiation at various levels for the 22 populations of $P$. stenoptera was characterized using hierarchical AMOVA within Arlequin 3.5 [33]. To calculate gene flow $(\mathrm{Nm})$ among populations, we also calculated $F_{\mathrm{ST}}$ based on neutral loci (i.e. excluding all outlier loci identified by Arlequin and BayeScan). The value of gene flow was estimated according to $1 / 4\left(1 / F_{\mathrm{ST}}-1\right)$. A total of 43 environmental variables (Additional files 2 and 3 ), including 11 temperature variables, 8 precipitation variables, 12 solar radiation variables, and 12 water vapor pressure variables, were downloaded from Worldclim (http://www.diva-gis.org/climate) from 1970 to 2000 at 2.5 arcmin resolution and further extracted using DIVA-GIS 7.5 [34]. The strongly correlated environmental variables with a Pearson correlation coefficient above 0.95 were eliminated. The correlation analysis was performed in SPSS 19 (SPSS Inc., Chicago, IL, USA). Thus, the remaining environmental variables were used for the subsequent RDA and environmental association analysis. After removing the strongly correlated environmental variables, 8 temperature variables (Bio1, Bio2, Bio3, Bio4, Bio5, Bio6, Bio8, and Bio9), 5 precipitation variables (Bio12, Bio13, Bio14, Bio15, and Bio18), 8 solar radiation variables (Sr1, Sr3, Sr5, Sr6, Sr7, Sr9, Sr10, Sr11), and 4 water vapor pressure variables (Wvp1, Wvp4, Wvp7, Wvp9) were retained. To infer the influence of the environmental variables to population genetic differentiation, we performed a constrained linear ordination analysis, RDA, in CANOCO 4.5 [35]. Here, allele frequencies per population (Additional file 4) were used as response variable and the remaining 30 uncorrelated environmental variables were used as explanatory variables.

To minimize the false positive rate, two methods were used to identify the mutual outlier loci for subsequent environmental association analysis. The first method is a hierarchical island model in Arlequin 3.5 [33]. The advantage of this approach is that it has better sensitivity to samples with common history and substructure. The program parameters used were as follows: 20,000 coalescent simulations, 100 simulated demes, and the number of simulated groups suggested by the results of STRUCTURE based on all loci. The loci with $F_{\mathrm{ST}} P$-value below 0.05 were considered as outlier loci, whereas those with total allele frequencies below 0.05 or above 0.95 were removed from the final 
results. The second method is a Bayesian method in BayeScan 2.0 [36]. The advantage of this approach is that it allows the population samples to have different amounts of genetic drift or different demographic histories [37]. The program parameters used were as follows: sample size of 5000, thinning interval of 10,20 pilot runs with 5000 run length, 50,000 burn-in iterations, and 10,000 prior odds. The loci with posterior probability over 0.76 were considered as outlier loci. To verify whether these loci are driven by environmental variables, environmental association analysis were performed using LFMM 1.2 [38]. This method based on latent factor mixture model can effectively avoid the misidentification of EAL caused by population history or population structure. The analysis was run with the following parameters: 10,000 sweeps, 1000 burn-in sweeps, and the number of latent factors from the results of STRUCTURE based on neutral loci (excluding all suspected outlier loci identified by Arlequin and BayeScan). The loci with $|\mathrm{z}|$ over 3 and $P$ below 0.001 were considered as EAL.

\section{Additional files}

Additional file 1: The outlier loci identified by Arlequin and BayeScan. (DOCX $31 \mathrm{~kb})$

Additional file 2: Fifty-five environmental variables used in this study. (DOCX $16 \mathrm{~kb}$ )

Additional file 3: Environmental variables for each location from the WorldClim database. (DOCX 26 kb)

Additional file 4: Allele frequencies of 1006 alleles for each population. (DOCX $253 \mathrm{~kb}$ )

\section{Abbreviations}

AFLPS: Amplified fragment length polymorphisms; AMOVA: Analysis of molecular variance; EAL: Environment-associated loci; ISSRs: Inter-simple sequence repeats; PCRs: Polymerase chain reactions; RDA: Redundancy analysis; SCOTs: Start codon targeted polymorphisms

\section{Acknowledgements}

We are grateful to Jie Yang and Cai-Yun Miao for help during the field work for collecting samples.

\section{Funding}

This work was supported by Natural Science Foundation of Henan Province (182300410039) for genotyping of this study, the National Natural Science Foundation of China (31770225) and Henan Agricultural University Science \& Technology Innovation Fund (KJCX2016A2) for field sampling, the Opening Project of Guangdong Provincial Key Laboratory of Plant Resources (PlantKF09) for writing the manuscript, the Funding Scheme of Young Backbone Teachers of Higher Education Institutions in Henan Province (2015GGJS-081) for all experimental materials of this study.

\section{Availability of data and materials}

The datasets supporting the conclusions of this article are included within the article and its additional files. Material samples are available from authors.

\section{Authors' contributions}

YLi conceived and designed the experiments, and wrote the paper; J-XL and $\mathrm{X}-\mathrm{HZ}$ performed the experiments and analyzed the data; YLiu, Z-HQ, X-XZ, $Y S$, and $L-Y J$ collected the samples and read the raw data. All authors read and approved the final version of the manuscript.

\section{Ethics approval and consent to participate}

No specific permits were required for $P$. stenoptera, all samples were collected by researchers following current Chinese regulations.

\section{Consent for publication}

Not applicable.

\section{Competing interests}

The authors declare that they have no competing interests.

\section{Publisher's Note}

Springer Nature remains neutral with regard to jurisdictional claims in published maps and institutional affiliations.

\section{Author details}

${ }^{1}$ Innovation Platform of Molecular Biology, College of Forestry, Henan Agricultural University, No.95, Wenhua Road, Zhengzhou 450002, China. ${ }^{2}$ Guangdong Provincial Key Laboratory of Plant Resources, School of Life Sciences, Sun Yat-Sen University, No.135, Xingang Xi Road, Guangzhou 510275, China.

Received: 10 August 2018 Accepted: 14 November 2018

Published online: 27 November 2018

References

1. Di Pierroa EA, Mosca E, González-Martínez SC, Binelli G, Neale DB, La Porta N. Adaptive variation in natural alpine populations of Norway spruce (Picea abies [L.] karst) at regional scale: landscape features and altitudinal gradient effects. Forest Ecol Manag. 2017:405:350-9.

2. Yang J, Miao CY, Mao RL, Li Y. Landscape population genomics of forsythia (Forsythia suspensa) reveal that ecological habitats determine the adaptive evolution of species. Front Plant Sci. 2017;8:481.

3. Rellstab C, Gugerli F, Eckert AJ, Hancock AM, Holderegger R. A practical guide to environmental association analysis in landscape genomics. Mol Ecol. 2015;24:4348-70

4. Li Y, Zhang XX, Mao RL, Yang J, Miao CY, Li Z, Qiu XY. Ten years of landscape genomics: challenges and opportunities. Front Plant Sci. 2017;8:2136

5. Stinchcombe JR, Hoekstra HE. Combining population genomics and quantitative genetics: finding the genes underlying ecologically important traits. Heredity. 2008;100:158-70.

6. Joost S, Bonin A, Bruford MW, Després L, Conord C, Erhardt G, Taberlet P. A spatial analysis method (SAM) to detect candidate loci for selection: towards a landscape genomics approach to adaptation. Mol Ecol. 2007;16:3955-69.

7. Sork VL, Aitken SN, Dyer RJ, Eckert AJ, Legendre P, Neale DB. Putting the landscape into the genomics of trees: approaches for understanding local adaptation and population responses to changing climate. Tree Genet Genomes. 2013;9:901-11.

8. Deng L, Liang Q, He X, Luo C, Chen H, Qin Z. Investigation and analysis of genetic diversity of diospyros germplasms using SCOT molecular markers in Guangxi. PLoS One. 2015;10:e0136510.

9. Elshire RJ, Glaubitz JC, Sun Q, Poland JA, Kawamoto K, Buckler ES, Mitchell SE. A robust, simple genotyping-by-sequencing (GBS) approach for high diversity species. PLoS One. 2011;6:e19379.

10. Miller MR, Dunham JP, Amores A, Cresko WA, Johnson EA. Rapid and costeffective polymorphism identification and genotyping using restriction site associated DNA (RAD) markers. Genome Res. 2007;17:240-8.

11. Sun $X$, Liu D, Zhang X, Li W, Liu H, Hong W, Jiang C, Guan N, Ma C, Zeng H, Xu C, Song J, Huang L, Wang C, Shi J, Wang R, Zheng X, Lu C, Wang X, Zheng $H$. SLAF-seq: an efficient method of large-scale de novo SNP discovery and genotyping using high-throughput sequencing. PLoS One. 2013;8:e58700.

12. Harris SE, Munshi-South J. Signatures of positive selection and local adaptation to urbanization in white-footed mice (Peromyscus leucopus). Mol Ecol. 2017;26:6336-50.

13. Keller SR, Chhatre VE, Fitzpatrick MC. Influence of range position on locally adaptive gene-environment associations in Populus flowering time genes. J Hered. 2018;109:47-58 
14. Miao CY, Li Y, Yang J, Mao RL. Landscape genomics reveal that ecological character determines adaptation: a case study in smoke tree (Cotinus coggygria Scop.). BMC Evol Biol. 2017;17:202.

15. Yang J, Li Y, Miao CY, Mao RL. Landscape genomics analysis of Achyranthes bidentata reveal adaptive genetic variations are driven by environmental variations relating to ecological habit. Popul Ecol. 2017;59:355-62.

16. Fu ZZ, Li YH, Zhang KM, Li Y. Molecular data and ecological niche modeling reveal population dynamics of widespread shrub Forsythia suspensa (Oleaceae) in China's warm-temperate zone in response to climate change during the Pleistocene. BMC Evol Biol. 2014;14:114.

17. Xu YM, Zhou MH, Shi YH, Hu XY, Yuan KK. Advance on the biological properties and resources utilization of Pterocarya stenoptera. J Northeast Fore Univ. 2002;30:42-8.

18. Orsini L, Mergeay J, Vanoverbeke J, De Meester L. The role of selection in driving landscape genomic structure of the waterflea Daphnia magna. Mol Ecol. 2013;22:583-601.

19. Hall LA, Beissinger SR. A practical toolbox for design and analysis of landscape genetics studies. Landsc Ecol. 2014;29:1487-504.

20. Ohsawa T, Ide Y. Global patterns of genetic variation in plant species along vertical and horizontal gradients on mountains. Glob Ecol Biogeogr. 2008;17:152-63.

21. Hickerson MJ, Carstens BC, Cavendar-Bares J, Crandall KA, Graham CH, Johnson JB, Rissler L, Victoriano PF, Yoder AD. Phylogeography's past, present and future: 10 years after Avise, 2000. Mol Phylogenet Evol. 2010:54:291-301.

22. Johnson MTJ, Prashad CM, Lavoignat M, Saini H. Contrasting the effects of natural selection, genetic drift and gene flow on urban evolution in white clover (Trifolium repens). P Roy Soc B-Biol Sci. 2018;285:20181019.

23. Wright S. Evolution in Mendelian populations. Genetics. 1931;16:97-159.

24. Xue XJ, Gao FZ, Wang RZ. The experiment report of different provenance of Pterocarya stenoptera. J Shandong Forest Sci Technol. 1991;79:27-30.

25. Collard BCY, Mackill DJ. Start codon targeted (SCOT) polymorphism: a simple, novel DNA marker technique for generating gene-targeted markers in plants. Plant Mol Biol Report. 2009;27:86-93.

26. Nei M. Analysis of gene diversity in subdivided populations. Proc Natl Acad Sci U S A. 1973;70:3321-3.

27. Vekemans $X$, Beauwens $T$, Lemaire $M$, Roldán-Ruiz I. Data from amplified fragment length polymorphism (AFLP) markers show indication of size homoplasy and of a relationship between degree of homoplasy and fragment size. Mol Ecol. 2002;11:139-51.

28. Pritchard JK, Stephens M, Donnelly P. Inference of population structure using multilocus genotype data. Genetics. 2000;155:945-59.

29. Evanno G, Regnaut S, Goudet J. Detecting the number of clusters of individuals using the software structure: a simulation study. Mol Ecol. 2005;14:2611-20.

30. Earl DA, vonHoldt BM. STRUCTURE HARVESTER: a website and program for visualizing STRUCTURE output and implementing the Evanno method. Conserv Genet Resour. 2012;4:359-61.

31. Jakobsson M, Rosenberg NA. CLUMPP: a cluster matching and permutation program for dealing with label switching and multimodality in analysis of population structure. Bioinformatics. 2007;23:1801-6.

32. Rosenberg NA. DISTRUCT: a program for the graphical display of population structure. Mol Ecol Notes. 2004:4:137-8.

33. Excoffier $L$, Lischer HEL. Arlequin suite ver 3.5: a new series of programs to perform population genetics analyses under Linux and windows. Mol Ecol Resour. 2010;10:564-7.

34. Hijmans RJ, Guarino L, Cruz M, Rojas E. Computer tools for spatial analysis of plant genetic resources data: 1. DIVA-GIS. Plant Genet Resour Newsl. 2001;127:15-9.

35. Ter Braak CJF, Smilauer P. CANOCO reference manual and CanoDraw for windows user's guide: software for canonical community ordination (version 4.5). New York: Microcomputer Power; 2002.

36. Foll $M$, Gaggiotti OE. A genome scan method to identify selected loci appropriate for both dominant and codominant markers: a Bayesian perspective. Genetics. 2008;180:977-93.

37. Manel S, Conord C, Després L. Genome scan to assess the respective role of host-plant and environmental constraints on the adaptation of a widespread insect. BMC Evol Biol. 2009;9:288.

38. Frichot $E$, Schoville $S D$, Bouchard $G$, Francois $\mathrm{O}$. Testing for associations between loci and environmental gradients using latent factor mixed models. Mol Biol Evol. 2013;30:1687-99.

Ready to submit your research? Choose BMC and benefit from:

- fast, convenient online submission

- thorough peer review by experienced researchers in your field

- rapid publication on acceptance

- support for research data, including large and complex data types

- gold Open Access which fosters wider collaboration and increased citations

- maximum visibility for your research: over $100 \mathrm{M}$ website views per year

At BMC, research is always in progress.

Learn more biomedcentral.com/submissions 